\title{
3D Simulation of Environmental Conflict Resolution in Intelligent Environments
}

\author{
Jaewook Lee \\ Department of Architectural Engineering, Sejong University, Seoul, Korea
}

\begin{abstract}
Owing to the advent of ubiquitous computing technologies, the concept of Intelligent Environments has emerged, as an alternative approach to overcome the limitation of the built environment. Intelligent Environments can be more responsive to userand context-specific human activities by automatically and dynamically modifying their settings without explicit human intervention. However, the conventional approach to the development of Intelligent Environments has mainly focused on the technical issues without paying much attention to the dynamic interrelationship between the user and the built environment. Therefore, differing or conflicting needs of multiple simultaneous users remain unresolved. The objective of this study is to present types of environmental conflicts and their resolution through agent collaboration and negotiation. For the demonstration and validation of the conflict resolution process, a set of hypothetical test cases is simulated in a 3D test environment. The result of the case simulations shows that the proposed approach is computationally feasible and applicable to the development of Intelligent Environments, and, furthermore, it can overcome the drawback of the conventional approach.
\end{abstract}

Keywords : Intelligent Environments, Environmental Conflicts, Conflict Resolution, Agent Collaboration, 3D Simulation

\section{INTRODUCTION}

Users' needs of the built environment tend to change over time due to the change of users (current and future users), activities (work, rest, etc.), or preferences (individual and group preferences). Rapoport (1990), in this sense, emphasizes the personalization of the environment as a way of presenting self through "taking possession, completing it, and changing it." However, as many researchers and practitioners point out (Alexander, 1964; Friedman, 1975; McLeod, 1997; Negroponte, 1975), the conventional built environment cannot fully accommodate ever-changing environmental needs due to the nature of the design process and its outcome, the built environment. Consequently this often leads to environmental conflicts among users, activities, and building components.

Corresponding Author: Jaewook Lee, Assistant Professor Department of Architectural Engineering, Sejong University 98 Gunja-Dong, Gwangjin-Gu, Seoul, 143-747, Korea

Tel: +82 234083836 e-mail : jaewook@sejong.ac.kr This work was supported by the faculty research fund of Sejong University in 2011

This is an Open Access article distributed under the terms of the Creative Commons Attribution Non-Commercial License (http://creativecommons. org/licenses/by-nc/3.0/) which permits unrestricted non-commercial use, distribution, and reproduction in any medium, provided the original work is properly cited.
To overcome this limitation, the concept of Intelligent Environments has been introduced with the advent of ubiquitous computing technologies including sensors, handheld mobile devices and powerful micro-computers (Weiser, 1993). Unlike conventional built environments, Intelligent Environments can support diverse user activities by automatically and dynamically adjusting their settings without explicit human intervention. Two major approaches to the development of Intelligent Environments include: (1) utilizing individual devices or systems to execute simple environmental control functions independently from other devices or systems (Kidd et al., 1999; Krogh et al., 2001; MIT Media Lab, 2011); and (2) utilizing multiple devices or systems to perform more complex control functions in collaboration with other devices or systems (Boman et al., 1998; Brumitt et al., 2000; Coen, 1998; Colley et al., 2001; Holmes et al., 2002; Mozer, 1998; Sharples et al., 1999; MIT Media Lab, 2011). However, these conventional approaches have mainly focused on the technical issues about the physical components without paying much attention to the dynamic interrelationship between the user and the built environment. Therefore, differing or conflicting needs of multiple simultaneous users remain unresolved. Particularly in an Intelligent Environment where multiple intelligent agents (or devices) collectively modify the setting of the environment (Jennings, 2000; Kulkarni, 2002; Russell \& Norvig, 2003; Sandhu, 2004; Vinyals, 2008; Weiss, 1999), environmental conflicts should be promptly identified and resolved for the consistency of environment-wide setting modification. 
This study aims to present types of environmental conflicts within Intelligent Environments and how these environmental conflicts can be resolved through agent collaboration and negotiation. To demonstrate and validate the process of the conflict resolution, a set of hypothetical test cases is simulated using a 3D game engine, Torque.

\section{TEST MODEL AND ENVIRONMENTAL CONFLICTS}

\section{(1) Test Model: A Multiagent-based Intelligent} Environment

\section{a. Structure of Test Model}

In the test model, individual building components (doors, furniture, lights, etc.) are represented as multiple intelligent agents that know how to modify the setting of the environment. They have the ability to perceive contextual changes of the environment and respond to them to support users' context-specific activities. These multiple agents construct a team-like organization and resolve environmental conflicts through collaboration and negotiation with other agents. The agent organization is built on a layered structure that includes three agent levels, arranged in a hierarchical structure (Figure 1).

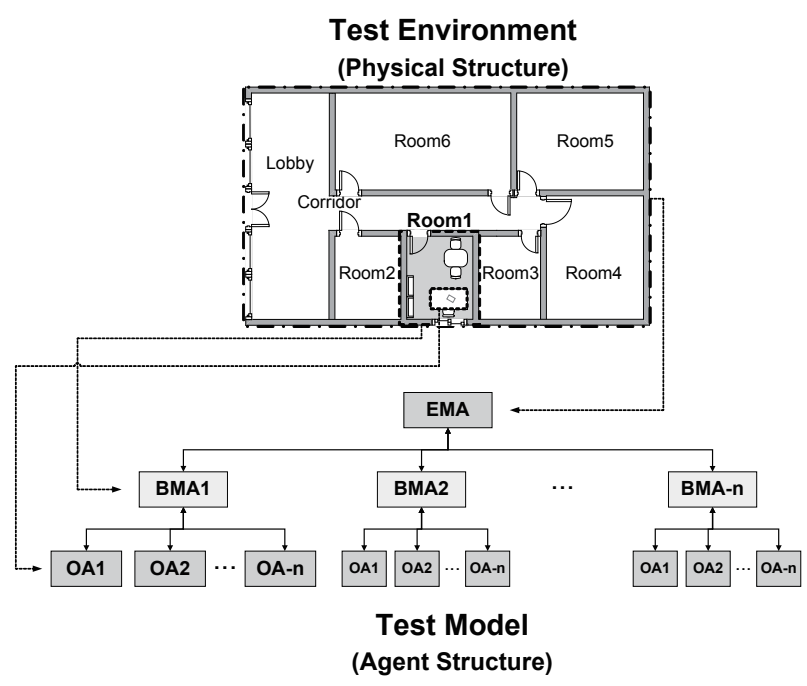

Figure 1. Layered Structure of Test Model

The three agent levels and their roles are: (1) Object Agent (OA) is the lowest level agent that directly controls the corresponding object to respond to activities of the user who occupies the object; and (2) Behavior Management Agent (BMA), as the intermediate agent in the hierarchy, controls the assigned zone (a room, a lobby, etc.) composed of a number of objects by handling conflicts between OAs; and (3) Environment Monitoring Agent (EMA) is the top level agent that controls the overall behavior of the whole environment by identifying the context of each zone and deals with zone-level conflicts (i.e., conflict between BMAs).

Two fundamental advantages of this approach are: (1) division of labor and individual autonomy can improve the efficiency of the overall performance of the Intelligent Environment by enhancing information processing; and (2) collaboration can overcome the limited knowledge and ability of individual agents, and can accomplish environment-wide modification by combining modifications executed by individual agents.

\section{b. Profile-based Setting Modification}

The setting modification of the test environment requires three types of profiles: (1) 'Object Profiles' contain the behavior description of individual objects, which directs the sensingresponding process of the objects according to the change of users and their activities; (2) 'User Profiles' encode users' environmental preferences including user ID, object IDs, property variables, and their values (e.g., lighting level, temperature, humidity, etc.). In a real environment, they can be stored in a RFID tag and read by objects wirelessly; and (3) 'Activity Profiles' include users' activity-specific preferences for the setting of the environment in consideration of types and/or times of activities. With these profiles, the test environment can identify users and their activities, and respond to them properly (Figure 2).

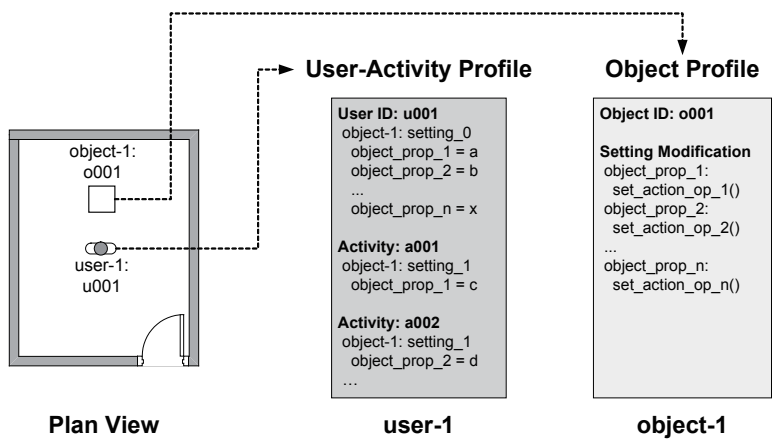

Figure 2. Plan View and Profiles

\section{(2) Types of Environmental Conflicts}

The term conflict is defined as "a breakdown in the standard mechanisms of decision-making so that an individual or group experiences difficulty in selecting an action alternative" (March \& Simon, 1958). Three main classes of conflicts are: (1) individual conflicts - conflicts in individual decision-making; (2) organizational conflicts - individual or group conflicts in organizations; (3) inter-organizational conflicts - conflict between organizations or groups. Individual conflicts generally arise when unacceptability, incomparability, or uncertainty exist in decisionmaking. In most cases of individual conflicts, the reaction of individuals to a conflict is to generate a search behavior for alternatives and evaluate their outcome before making a choice (Boulding, 1962; Tessier, 2001; Tidwell, 1998). In contrast, the other types of conflicts mostly arise due to the goal difference between parties. Although some conflicts may result in positive outcomes, conflict is generally admitted as an unfavorable situation for the parties involved. Particularly, conflict may lead to a deadlock situation where no further progress of achieving the goal(s) is possible.

Similar to human organizations, in the built environment, as a mixture of users and objects, two different types of environmental conflicts can be observed: object-side conflicts between physical objects; and user-side conflicts between multiple simultaneous users. In conventional built environments, the resolution of these conflicts is left to human occupants in the form of the on-going actions (turning on lights, opening windows, re-arranging the furniture, even remodeling the building when more extensive changes are needed). However, in Intelligent Environments, an effort to resolve environmental conflicts should be made by the intelligent agents, thereby off-loading decisions and actions 
from the human occupants. In the test environment, the object agents search for potential settings that can transform the current state of the environment to a desired one, and identify possible environmental conflicts and resolve them before applying the searched settings. The following describes the details of each conflict type in the test environment.

\section{a. Object-Side Conflicts}

This type of conflict results from perception or goal differences between object agents $(\mathrm{OAs})$. An OA, as a spatiotemporally and rationally bounded entity, can only perceive a (small) part of the environment about which the OA has subjective knowledge as well as limited reasoning capacity. As a result, different OAs may interpret the same user activity differently. Moreover, each OA has its own goal which may be different from that of other OAs. These perception and goal differences are a major source of object-side conflicts.

\section{b. User-Side Conflicts}

When two or more users simultaneously exist in the same environment, there can be differences in their preference over the settings of the environment. That is, an overall setting of the room that one user prefers may conflict with that of other user(s). This preferential difference between users may result in user-side conflicts in the test environment.

Similarly, whereas a single user normally performs a single activity for a certain time period, two or more users may perform different activities in the same environment at the same time, which may also lead to a user-side conflict. For instance, in an office, the lighting and sound preference for the resting activity of one user may conflict with the preference for the working activity of another user. As such user-sides conflicts arise due to the preferential difference between users, and may not be easily predicted in advance.

\section{TOROUE GAME ENGINE (TGE) AND SIMULATION OVERVIEW}

\section{(1) Torque Game Engine (TGE)}

Torque is a commercial game engine suitable for creating $3 \mathrm{D}$ games including action, fighting, role-playing, and simulation games. Torque Game Engine (TGE) was originally developed in 2001 by Dynamics for Tribes 2, a FPS (First Person Shooter), but had been re-engineered to be a general game-development kit for multiple platforms (e.g., Windows, Mac OS, and Linux) (GarageGames, 2011). TGE provides 3D rendering, networking, scripting, world-editing, character animation, and GUI (Graphical User Interface) creation for game developers. In addition to game development, TGE has potential in building MUVE (Multi-User Virtual Environments) (IJsselsteijn \& Riva, 2003; Kalay \& Grabowicz, 2006) (Figure 3), and it has been used for environmental design researches (Moloney et al., 2003; O'Connor et al., 2005).

In 3D games developed with TGE, users are typically represented as an avatar that can navigate the game environment. The avatar can also interact with objects, other avatars, and NPCs (NonPlayer Characters) within the environment. TGE's built-in server/ client network allows real-time communication over the Internet between the users who share the same environment. In the current version of Torque, user communication is typically done through a chat window. In consideration of its ready-to-use and modifiable functions, TGE was selected to simulate the test cases. For the case simulations, TGE's TorqueScript is used in conjunction with the modification of the $\mathrm{C}++$ source code.

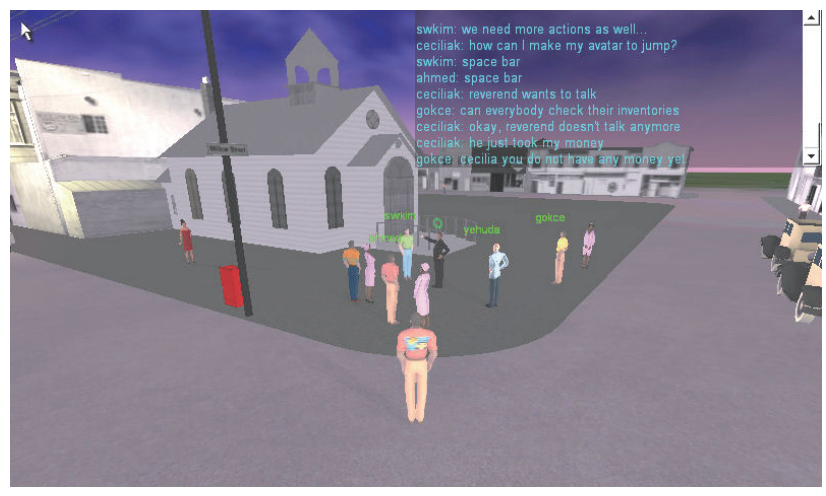

Figure 3. West Oakland Project (Kalay \& Grabowicz, 2006)

\section{(2) Simulation Overview}

The test environment begins with a minimal setting that contains a single user and object. Thereafter, as the simulation progresses, it changes to include an additional user and/or object. Each of the four test cases simulates a specific setting modification by a single agent or multiple agents that comprise the test environment.

Table 1. Simulation Configurations and Types of Agent Behavior

\begin{tabular}{c|c|c|c}
\hline Case & Users & Objects & Types of Agent Behavior \\
\hline C.1 & 1 & 1: Chair & Perception \\
\hline C.2.1 & 1 & $\begin{array}{c}\text { 2: Chair and } \\
\text { Lighting }\end{array}$ & Collaboration \\
\hline C.2.2 & 1 & $\begin{array}{c}\text { 2: Chair and } \\
\text { Desk }\end{array}$ & $\begin{array}{c}\text { Conflict Resolution: } \\
\text { Coordination }\end{array}$ \\
\hline C.3 & 2 & 1: Sofa & $\begin{array}{c}\text { Conflict Resolution: } \\
\text { Group - Activity Profile }\end{array}$ \\
\hline C.4 & 2 & $\begin{array}{c}\text { 2: Sofa and } \\
\text { Table }\end{array}$ & $\begin{array}{c}\text { Conflict Resolution: } \\
\text { Coordination \& Group - } \\
\text { Activity Profile }\end{array}$ \\
\hline
\end{tabular}

The first simulation shows the simple sensing and acting ability of OAs through the interaction between one user and one object. The second simulation is for testing agent coordination using one user and two objects. The major concerns of this simulation are an object-side setting conflict and its resolution. The third simulation, which contains two users and one object, exhibits the process of resolving a user-side, preferential conflict. Finally, the last simulation deals with a case that generates both an object- and user-side conflict in the test environment. In this simulation, the BMA of the test environment combinationally utilizes agent coordination and conflict resolution to resolve both the object- and user-side conflict simultaneously. Table 1 shows the overall configuration and major agent behavior of the simulation cases.

\section{SIMULATION OF CONFLICT RESOLUTION}

The simulation program consists of two components: a 3D 
viewer and a status window. The $3 \mathrm{D}$ viewer shows the current test environment that includes object(s) and user(s). For each case simulation, the viewer displays a different environment. The status window is for displaying the detailed condition of the test environment in a text format. If any environmental changes (setting, conflict, resolution, etc.) occur in the environment, they will be displayed on the status window. In the case simulations, each user is a preprogrammed NPC that interacts with the object(s).

\section{(1) Case 1: Agent Sensing and Acting}

In the test environment, OAs initially sense the user and his/her activity before acting to modify their setting. Thus, this simulation tests OAs' essential sensing and acting ability.

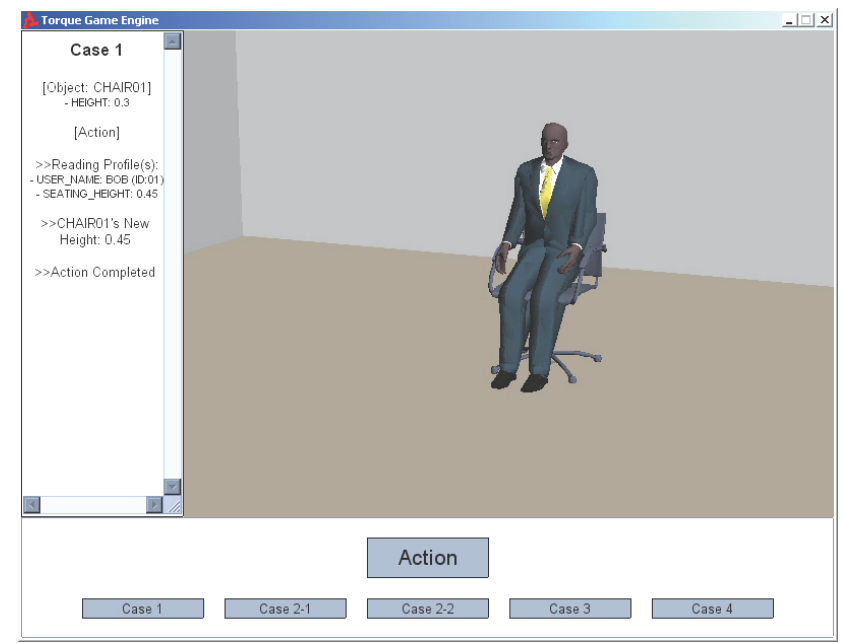

Figure 4. Case 1: Agent Sensing and Acting

At the initial state of the simulation, a user, Bob, is away from an object, chair, in the environment. In this state, the chair OA does not detect the current user, Bob, yet.
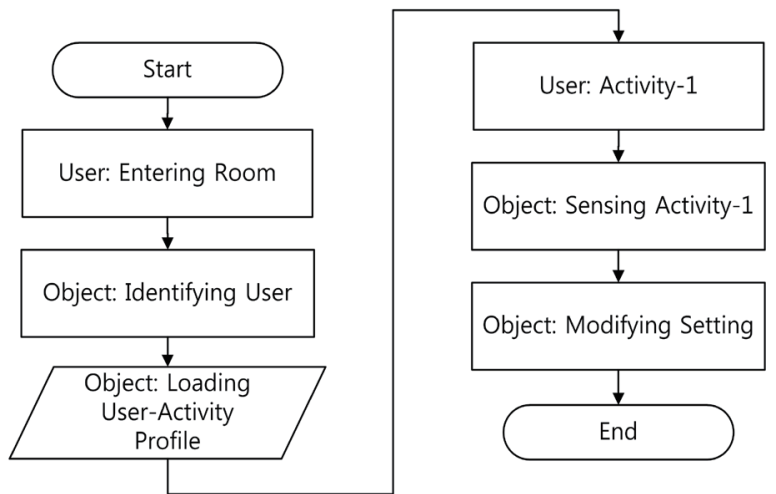

Figure 5. Object Profile - Sensing Function

When the "Action" button is clicked, Bob sits on the chair. At the same time, the chair OA detects Bob and loads his User-Activity Profile that includes his bodily data (standing height, sitting height, etc.), user activities (working, resting, etc.), and corresponding chair's properties (height, temperature, etc.). At this moment, the chair is ready to respond to the change of Bob's activity.

As Bob initiates his "Working" activity, the chair OA senses this activity and modifies its properties as described in the loaded profile (Figure 4). Figure 5 shows the sensing function that is a part of the chair's Object Profile.

\section{(2) Case 2: Agent Collaboration and Resolution of Object- Side Conflicts}

This case simulation comprises two sub-cases. While the first sub-case focuses on the agent collaboration of the test model, the second one is for testing the resolution of object-side conflicts.

\section{a. Case 2.1: Agent Collaboration}

Unlike the previous case simulation, the test environment of this simulation contains one user, Bob and two objects, a chair and lighting (Figure 6).

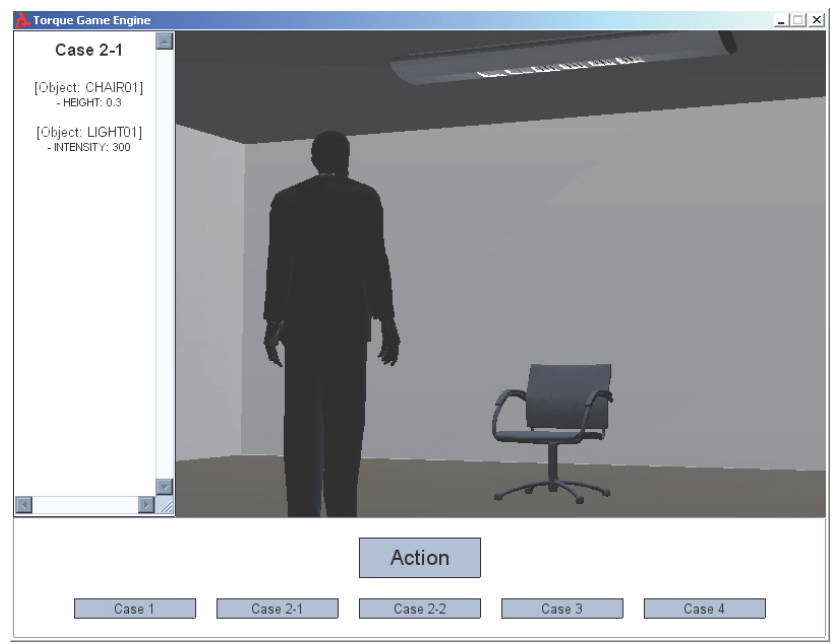

Figure 6. Case 2.1: Initial State

Here, the chair object is directly associated with Bob's activity, but the lighting object is indirectly associated with the activity. Therefore, to properly set its illumination level, the lighting OA has to know Bob's current activity from the chair OA through agent communication.

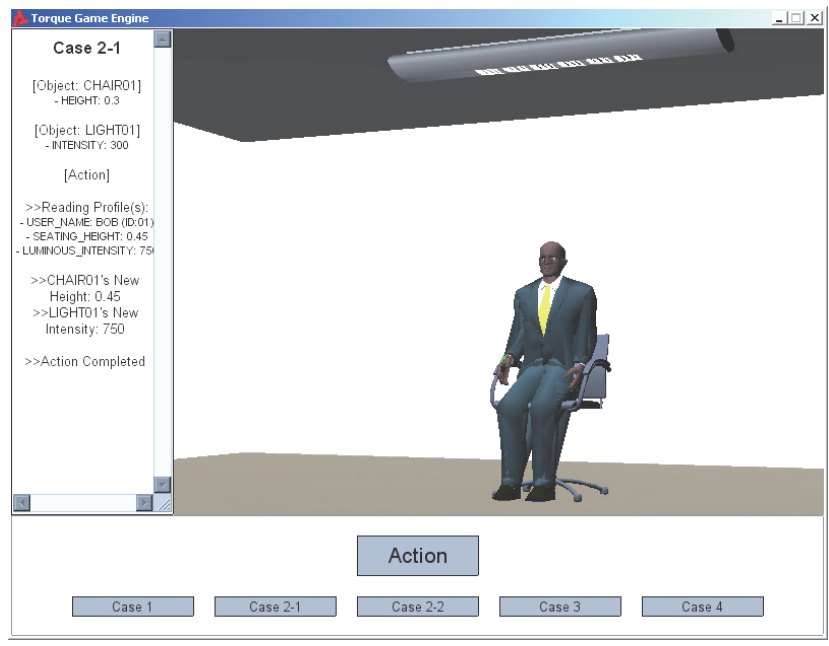

Figure 7. Case 2.1: Agent Collaboration

Once the chair OA identifies Bob's "Working" activity, it transmits this activity data to the lighting OA. Then the lighting OA searches for the corresponding illumination level in Bob's User-Activity 
Profile and applies it for Bobs "Working" activity received from the chair OA (Figure 7).

Figure 8 shows the lighting OA's sensing function that enables this message transmission. After the execution of the function, the lighting OA identifies Bob's current activity, "Working," for changing the lighting's illumination. As such, through this agent collaboration, the lighting OA can overcome its perceptual limitation.
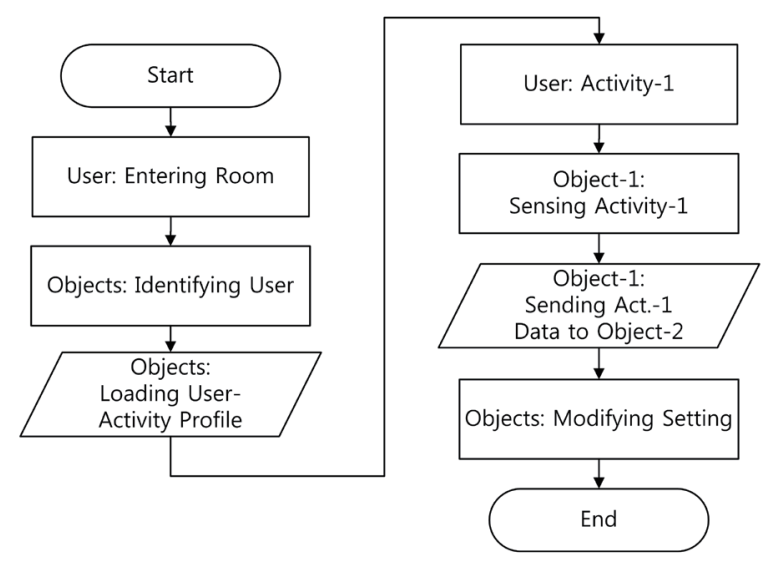

Figure 8. Agent Collaboration - Messaging Function

\section{b. Case 2.2: Resolution of Object-Side Conflicts}

In the test environment, two objects (i.e., a chair and desk) are directly associated with the user, Bob, who is "Resting." However, while the chair OA correctly reasons Bob's activity as "Resting," the desk OA reasons the same activity as "Working." This perceptual difference raises an object-side conflict (i.e., Resting $\neq$ Working). In this simulation, when the object-side conflict arises, the simulation program interrupts the process and displays a message, "Object-Side Conflict Detected." In addition, the status window shows the reason of the conflict and conflicting objects (Figure 9).

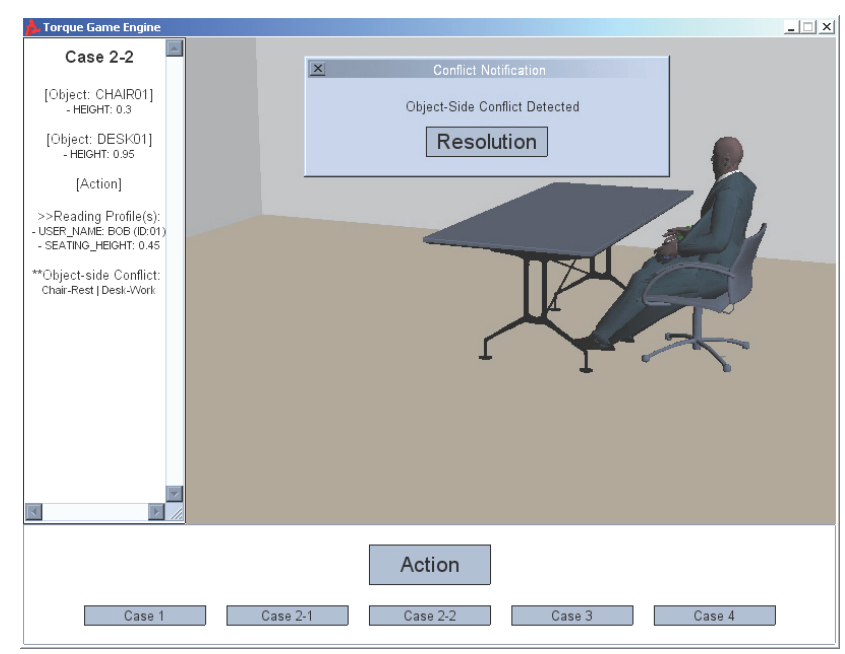

Figure 9. Case 2.2: Object-Side Conflict

The resolution of the object-side conflict between the chair and desk OA involves the BMA of the test model. After the "Resolution" button is pressed, the BMA detects the conflict, and, based on the Bob's use-history data store in the database, selects the chair OA's "Resting" percept as a correct one. This decision passes to the conflicting OAs (i.e., the chair and desk OA) and then the OAs modify the object setting for Bob's "Resting" activity.

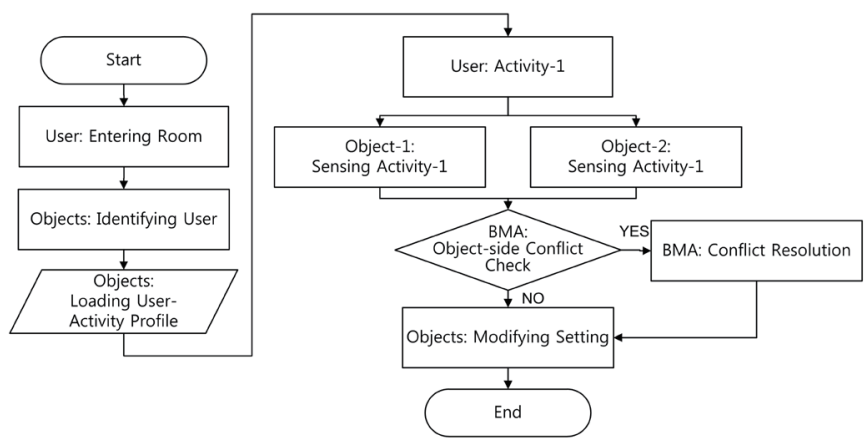

Figure 10. BMA - Resolution Function for Object-Side Conflicts

Figure 10 shows the resolution function for object-side conflicts, which is coded in the BMA's agent program. The main operation of this function is to equalize differing percepts between individual OAs.

\section{(3) Case 3: Resolution of User-Side Conflicts}

This simulation tests the resolution of user-side conflicts from users' preferential difference. The test environment includes one object, a sofa, and two users, Bob and Nancy. In the initial state, Bob and Nancy are standing beside the sofa, and as the simulation starts, they sit on the sofa.

At this moment, the sofa OA perceives the both Bob and Nancy, and loads their individual User- Activity Profiles. This means that the two different User-Activity Profiles are simultaneously active. Because Bob's preferred setting properties (height etc.) of the sofa are different from Nancy's, a user-side conflict occurs.

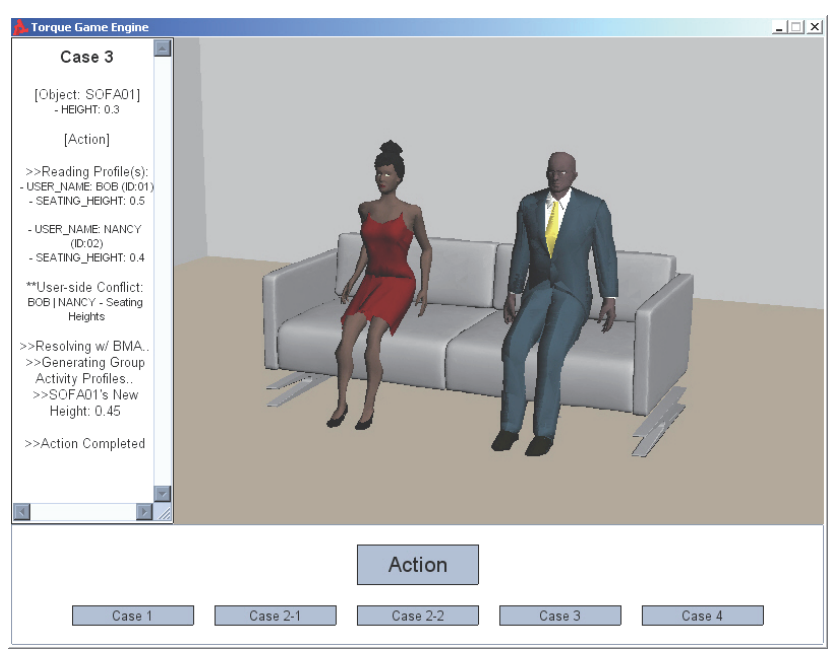

Figure 11. . Case 3: User-Side Conflict

To resolve this user-side conflict between the two users, the BMA creates a Group-Activity Profile that combines the individual UserActivity Profiles. In this simulation, the height property of the sofa is computed by averaging the individual height properties from the two profiles, and saved in the Group-Activity Profile. As the final state in Figure 11, the sofa OA modifies the height by applying the computed height value from the Group-Activity Profile. Figure 12 shows the BMA's resolution function for the user-side conflict. 


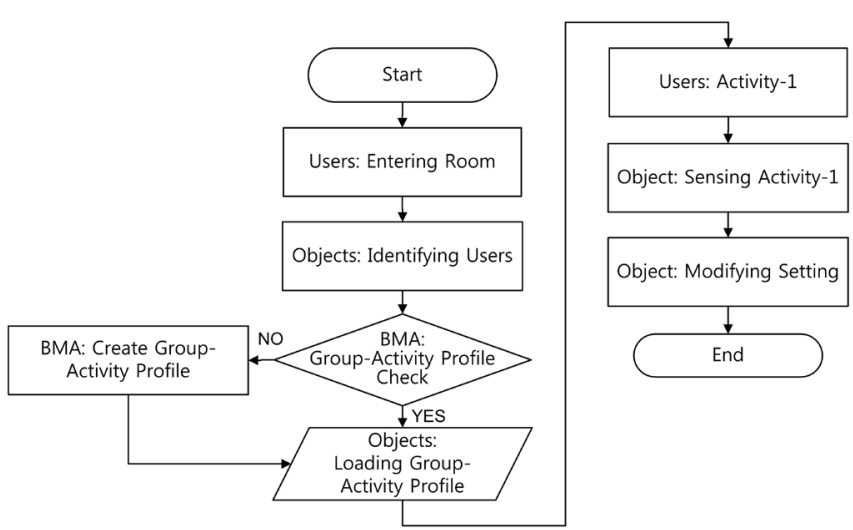

Figure 12. BMA - Resolution Function for User-Side Conflicts

\section{(4) Case 4: Resolution of Object- and User-Side Conflicts}

This is the most complex test case that simulates a combined conflict resolution for both an object- and user-side conflict in the test environment. It includes two users (i.e., Bob and Nancy) and two objects (i.e., a sofa and table). In the simulation, the object-side conflict results from the perceptual difference between the sofa and table OA - the sofa OA perceives Nancy's activity as "Resting," but the table OA perceives the same activity as "Working." Whereas, a user-side conflict arises from the sofa's setting modification because of the preferential difference between Bob and Nancy - the preferred property values of the sofa in Bob's User-Activity Profile are different from those in Nancy's profile.

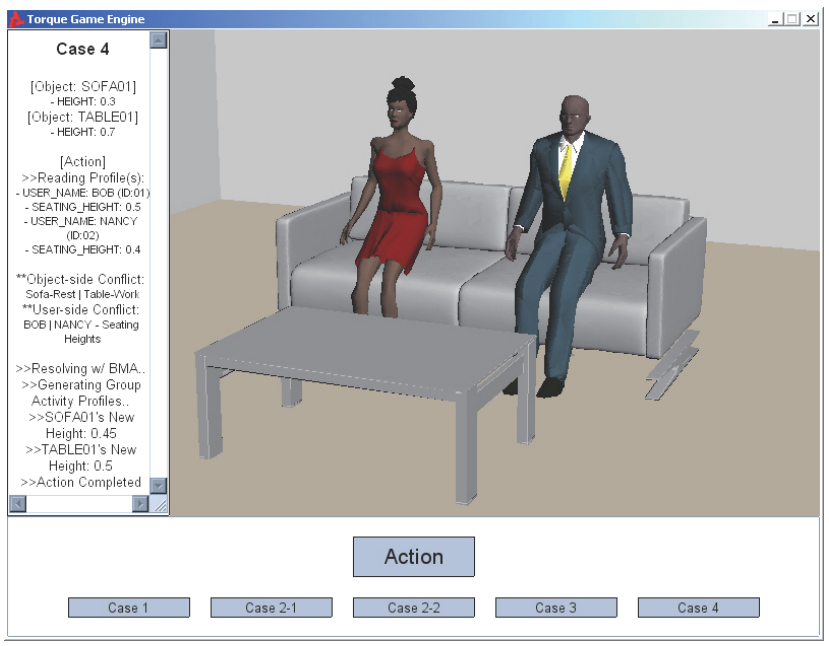

Figure 13. Case 4: Object- \& User-Side Conflict

For the resolution of both the object- and user-side conflict, the BMA of the test environment combinationally utilizes agent coordination and a Group-Activity Profile in the resolution process. First, the BMA generates a Group-Activity Profile based on Bob's and Nancy's User-Activity Profile, like Case 3, to resolve the userside conflict. Second, the BMA coordinates the sofa and table OA to correct the table's wrong percept, from "Working" to "Resting" as in Case 2.2. Thereby, the sofa and table OA are able to modify the object setting accordingly (Figure 13).

To enable this combined resolution process, the resolution function of the BMA requires revision to include the resolution of both object- and user-side conflicts as shown in Figure 14.

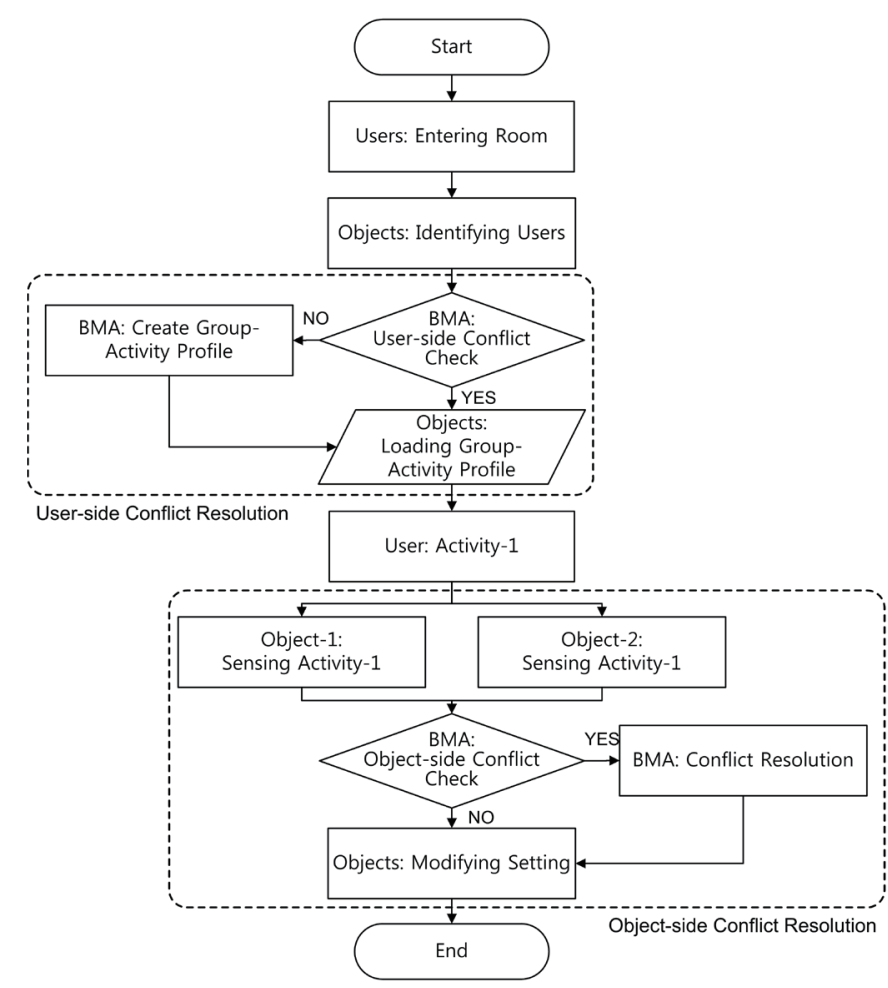

Figure 14. BMA - Resolution Function for User- \& Object-Side Conflicts

\section{CONCLUSION AND FUTURE WORK}

This study proposes a layered multiagent approach that can efficiently endow an environment with intelligence by organizing agents and controlling their behaviors. In the proposed model of Intelligent Environment, each building component is represented as an intelligent or smart agent that knows how to behave given any activity of any user. It has the ability to perceive changes of user activities in the environment and adjust its setting according to the changes through agent collaboration and conflict resolution .

To test the collaboration and resolution of environmental conflicts by the intelligent agents, four case simulations are exhibited. Each of them deals with a specific agent behavior in relation to the user activity in the environment. In the case simulations, as human designers solve a design problem by communicating and resolving conflicts with each other, OAs in the proposed model modify the setting of the environment through the communication and collaboration with other OAs as well as the BMA, a higher-level agent.

The case simulations demonstrate that agents' user identification, activity sensing, and communication are essential for building Intelligent Environments. Moreover, any object or user conflicts are required to be resolved in a timely fashion to minimize users' burden on controlling the environmental setting, and maximize their satisfaction.

However, these case simulations are based on a set of simplified hypothetical cases with a limited number of users and objects, so implementing the proposed approach in real-world Intelligent Environments will require more realistic case studies with more users and objects. Moreover, further research and experiment with physical objects and devices are needed. 
Also worth mentioning is that the test case simulated in this study do not include the control process by the Environment Monitoring Agent (EMA), the highest level agent of the test model. This is because the primary role of EMA is to control multiple rooms or zones that are spatiotemporally interconnected, but the current technology is not yet ready to implement this concept. Nevertheless, the EMA is an important component to realize building-level Intelligent Environments in the future, and thus it requires more conceptual and practical studies.

\section{REFERENCES}

Alexander, C. (1964). Notes on the synthesis of form. Cambridge, Massachusetts: Harvard University Press.

Boman, M., Davidsson, P., Skarmeas, N., Clark, K., \& Gustavsson, R. (1998). Energy Saving and Added Customer Value in Intelligent Buildings (Nawana and Ndumu Eds.), PAAM98, pp. 505-517.

Boulding, K. E. (1956). The image; knowledge in life and society. Ann Arbor: University of Michigan Press.

Brumitt, B., Meyers, B., Krumm, J., Kern, A., \& Shafer, S. A. N. (2000). EasyLiving: Technologies for Intelligent Environments. In Proceedings of the 2nd International Symposium on Handheld and Ubiquitous Computing, pp. 12-29.

Coen, M. H. (1998). A prototype intelligent environment, Proc. of 1st International Workshop on Cooperative Buildings (CoBuild 98).

Colley, M., Clarke, G., Hagras, H., Callaghan, V., \& PoundsCornish, A. (2001). Intelligent inhabited environments: Cooperative robotics \& buildings. Proc. of 32nd International Symposium on Robotics, Seoul, Korea.

Friedman, Y. (1975). Toward a scientific architecture. The MIT Press. Cambridge, Massachusetts.

GarageGames (2011). http://www.garagegames.com/

Holmes, A., Duman, H., \& Pounds-Cornish, A. (2002). The idorm: Gateway to heterogeneous networking environments, Proc. of International ITEA Workshop on Virtual Home Environments.

IJsselsteijn, W. \& Riva, G. (2003). Being There: The experience of presence in mediated environments, Being There: Concepts, effects and measurement of user presence in synthetic environments (G. Riva, F. Davide, \& W.A IJsselsteijn Eds.), Ios Press, Amsterdam.

Jennings, N. R. (1999). Agent-oriented software engineering. Proc. of 12th International Conference on Industrial and Engineering Applications of AI, Cairo, Egypt.

Kalay, Y. E. \& Grabowicz, P. (2006). Virtual Preservation of Seventh Street's 1950s Jazz Scene, 3D Visualization in the Arts Network, http://3dvisa.cch.kcl.ac.uk/paper_kalay.html

Kidd, C.D., Orr, R.J., Abowd, G., Atkeson, C.A., Essa, I., MacIntyre, B., Mynatt, E., Starner, T. \& Newstetter, W. (1999). The Aware Home: A Living Laboratory for Ubiquitous Computing Research. In Proceeding of 2nd International workshop on Cooperative Buildings (CoBuild99), Lecture Notes in Computer Science, 1670, Springer-Verlag, Berlin, pp. 191-198.

Krogh, P. \& Grønbæk, K. (2001). Roomware and intelligent buildings - objects and buildings become computer interfaces. In Proceedings of Architectural Research and Information Technology, Århus School of Architecture.
Kulkarni, A. (2002). A reactive behavioral system for the intelligent room. Technical report, MIT AI Lab.

March, J. S. \& Simon, H. A. (1958). Organizations. New York: Wiley.

McLeod, M. (1997). Henri Lefebvre Critique of Everyday Life: An Introduction. Architecture of the Everyday (S. Harris \& D. Berke Eds.), New York: Princeton Architectural Press.

Moloney, J., Amor, R., Furness, J., \& Moores, B. (2003). Design Critique Inside a Multi-Player Game Engine, In Proceedings of the CIB W78 Conference on Construction IT Bridging the Distance, Waiheke Island, New Zealand, 23-25 April, pp. 255262.

Mozer, M. C. (1998). The neural network house: An environment that adapts to its inhabitants. Proc. of AAAI Spring Symposium on Intelligent Environments, AAAI Press, pp. 110-114.

Negroponte, N. (1975). Soft architecture machines. MIT Press.

O'Connor, A., Stock, C., \& Bishop, I. (2005). SIEVE: An Online Collaborative Environment for Visualising Environmental Model Outputs, In Proc. of International Congress on Modeling and Simulation, Melbourne, December 12-15.

Rapoport, A. (1990). The Meaning of the Built Environment. The University of Arizona Press.

Russell, S. \& Norvig, P. (2003). Artificial Intelligence: A Modern Approach (2nd Edition). Prentice Hall Series on Artificial Intelligence, New Jersey.

Sandhu J. S., Agogino, A. M., \& Agogino, A. K. (2004). Wireless sensor networks for commercial lighting control: decisionmaking with multi-agent systems. Proc. of the AAAI-04 workshop on sensor networks, pp. 88-92.

Sharples, S., Callaghan, V., \& Clarke, G. S. (1999). A self-learning multi-agent architecture for intelligent building monitoring and control. International Sensor Review Journal, 19:1.

Streitz, N., Geißler, J., and Holmer, T. (1998). Roomware for cooperative buildings: Integrated design of architectural spaces and information spaces, Proc. of CoBuild 98.

Tessier, C., Chaudron, L., \& Muller, H. J. (Eds.) (2001). Conflicting Agents. Kluwer Academic Pub.

The Media Lab (2011). http://www.media.mit.edu/research/

Tidwell, A. (1998). Conflict Resolved?: A Critical Assessment of Conflict Resolution. New York: Pinter.

Vinyals, M., Rodrguez-Aguilar, J. A., \& Cerquides, J. (2008). A survey on sensor networks from a multi-agent perspective. Proc. of the second international workshop on agent technology for sensor networks (ATSN-08).

Weiser, M. (1993). Some computer science problems in ubiquitous computing. Communications of the ACM 36, No. 7, pp. 74-83.

Weiss, G. (Eds.) (1999). Multiagent systems: A modern approach to distributed artificial intelligence. Cambridge, Mass.: MIT Press.

(Date of Submission : 2009.5.27) 